\title{
927 WILMS TUMOR REVEALS DNA REPAIR GENE HYPER- EXPRESSION IS LINKED TO LACK OF IMMUNE INFILTRATION
}

${ }^{1}$ Emily Higgs*, ${ }^{2}$ Riyue Bao, ${ }^{1}$ Ken Hatogai, ${ }^{1}$ Thomas Gajewski. 'University of Chicago, Chicago, IL, USA; ${ }^{2}$ University of Pittsburgh, Pittsburgh, PA, USA

Background A $\mathrm{T}$ cell-rich tumor microenvironment has been associated with improved clinical outcome and response to immune checkpoint blockade therapies in several adult cancers. Understanding the mechanisms for lack of immune cell infiltration is critical for expanding immunotherapy efficacy in the clinic. To gain new insights into the mechanisms of poor tumor immunogenicity, we turned to pediatric cancers, which are generally unresponsive to checkpoint blockade.

Methods RNAseq and clinical data were obtained for Wilms tumor, rhabdoid tumor, osteosarcoma, and neuroblastoma from the Therapeutically Applicable Research to Generate Effective Treatments (TARGET) database, and adult cancers from TCGA. Using an 18-gene tumor inflammation signature (TIS) representing activated CD8 $+\mathrm{T}$ cells, we identified genes significantly anti-correlated with the signature. Immunofluorescence was performed on metastatic melanoma samples for CD8, MSH2, and the tumor cell marker SOX10, and analyzed for relationship to anti-PD-1 efficacy.

Results Among the four pediatric cancers, we observed the lowest TIS scores in Wilms tumor. Wilms tumors demonstrated significantly lower $\mathrm{T}$ cell inflammation signatures than matched normal kidney samples, other pediatric tumor samples, and adult kidney tumor samples. Pathway analysis identified multiple types of DNA repair were upregulated in Wilms tumor and a score generated from the top 50 DNA repair genes strongly anti-correlated with TIS. This striking negative association was also observed in most adult tumor types. The anti-correlation was found to be independent of tumor mutation burden, suggesting that high expression of DNA repair pathway machinery may restrict tumor immunogenicity by mechanisms beyond prevention neoantigen accumulation. MSH2 was one of the top DNA repair genes identified from the Wilms tumor analysis and was confirmed to have a strong anti-correlation with TIS in melanoma samples from TCGA. Immunofluorescence from an independent cohort of metastatic melanoma patients revealed a significant negative correlation between $\mathrm{CD} 8+\mathrm{T}$ cell numbers and $\mathrm{MSH} 2+\mathrm{SOX} 10+$ tumor cell numbers. Additionally, non-responders to anti-PD-1 immunotherapy had significantly higher numbers of $\mathrm{MSH} 2+$ SOX10+ tumor cells than responders.

Conclusions Increased tumor expression of DNA repair genes is associated with a less robust immune response in Wilms tumor, and this was also observed in the majority of TCGA tumor types. Surprisingly, the negative relationship between DNA repair score and TIS remained strong across TCGA when correcting for mutation count, indicating a potential role for DNA repair genes outside of preventing the accumulation of mutations. Strategies targeting DNA repair pathways could be considered as new therapeutic interventions to transform non-T cell-inflamed tumors into immune-responsive tumors.

Ethics Approval The study obtained ethics approval under IRB protocol 15-0837.

http://dx.doi.org/10.1136/jitc-2021-SITC2021.927 